Radiologe 2010 · 50:426-426

DOI 10.1007/s00117-010-1984-y

Online publiziert: 21. Februar 2010

๑) Springer-Verlag 2010

C. Schueller-Weidekamm $\cdot$ H. Imhof

Abteilung für Neuroradiologie und muskuloskelettale Radiologie, Klinik für Radiodiagnostik, Medizinische Universität Wien, AKH, Wien

\title{
Die Sportmedizin im Blickfeld der Radiologie
}

\section{Von der Verletzung zur Diagnose}

ketten („kinetic chains“) bieten hierfür die Basis. Der hohe Anteil an MRT-Abbildungen in den einzelnen Beiträgen dieser Ausgabe weist schon darauf hin, dass die MRT einen zentralen Stellenwert bei der Bildgebung von Sportverletzungen besitzt. Mit einer korrekt durchgeführten MRT-Untersuchung sollte die Anzahl der diagnostischen Arthroskopien gesenkt werden und der Anteil der therapeutischen Arthroskopien steigen.

Das Konzept der muskulotendinoossären Bewegungsketten wurde in der Sportmedizin entwickelt und ist hilfreich für den Nachweis pathologischer Veränderungen an allen relevanten Strukturen des Bewegungsapparats. Das Knochenmarködem z. B. ist ein wichtiger ,fingerprint “ für die Erkennung der Pathologien bei komplexen Verletzungsmustern und richtet das Augenmerk auf externe und interne Impingementsyndrome oder Zugkräfte, die über muskulotendinöse Strukturen auf den Knochen einwirken.

Dieses Schwerpunktheft von „Der Radiologe" richtet sich an Ausbildungs- und Fachärzte der Radiologie und an interessierte Leser, die ihre Kenntnisse im dem Gebiet der Sportmedizin auffrischen und vertiefen möchten. Populäre Sportarten wie Fußball spielen, Laufen, Mountainbiken oder Überkopfsportarten werden bzgl. ihrer akuten und chronischen Verletzungsgefahren, der radiologischen $\mathrm{Di}$ agnostik und therapeutischer Möglichkeiten sowie Prävention von Verletzungen abgehandelt.

Wir danken allen Autoren für ihre Beiträge,
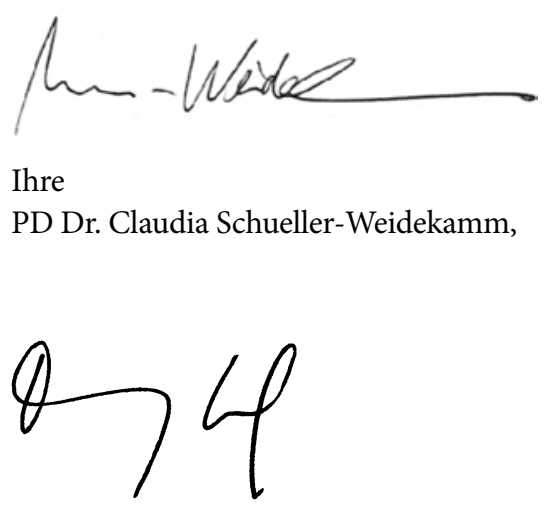

Ihr

Prof. Dr. Herwig Imhof 